\title{
Methods for broadband signals mutual time delays estimating enhancement
}

\author{
Ilya Grin $^{1, *}$ and Oleg Morozov ${ }^{1}$ \\ ${ }^{1}$ Lobachevsky State University of Nizhny Novgorod, Faculty of Physics, 603950 Nizhny Novgorod, \\ Russia
}

\begin{abstract}
This paper considers methods for estimating the mutual time delay of broadband signals recorded by satellites based multi-position systems for determining the location of a radiation source. All methods considered are based on modified algorithms for calculating the ambiguity function. The presented algorithms are based on the extraction of narrowband channels from the studied signals and their further optimal processing. The reliability criterion for mutual time delay estimation by the presented methods was evaluated. Based on the results and analysis of computational efficiency, viability of methods considered and their modifications was determined.
\end{abstract}

One of the most relevant applications of the radio sources location determining methods is their use for location and navigation problems solving based on multi-position space-based systems. Development of passive satellite multi-position systems for radio communication and navigation, which are operating under conditions of a priori uncertainty in signal parameters, has a particular interest.

The range-difference method is one of the most common methods for radio sources location determining, which involves the problem of estimating the propagation mutual time delays of the emitted signal distorted copies. Signals propagate through different channels under conditions of a low signal to noise ratio [1]. Modern communication systems use broadband and ultra-wideband signals, which can increase the stability of data transmission to noise, as well as improve the reliability of information transmission channels in difficult conditions of signal propagation.

Traditionally, the mutual time delay estimating problem is solved using correlation methods [2]. In particular, in the presence of a frequency shift of the spectra of the received signals due to the Doppler Effect, time delays are estimated based on the calculation and analysis of the mutual uncertainty function of the received signals [3]:

$$
A(\tau, \Delta f)=\int_{-\infty}^{+\infty} s_{1}(t) \cdot s_{2}^{*}(t+\tau) \exp (-j 2 \pi \Delta f t) d t
$$

The position of the main maximum of the mutual uncertainty function (1) allows us to determine the mutual signal delay and Doppler frequency shift:

* Corresponding author: grin.ilya@nifti.unn.ru 


$$
\left(\tau^{*}, \Delta f^{*}\right)=\arg \max _{\tau, \Delta f}|A(\tau, \Delta f)| .
$$

If the band of the received signal $B<<f_{0}$ ( $f_{0}$ is the carrier frequency), then the signal is narrowband (for example, phase-shifted signals used in GPS protocols). In that case the scaling of the spectrum can be neglected in comparison with the Doppler frequency shift and compensation of this shift can be made by direct mutual uncertainty function calculation (2). In the case when the signals are broadband $\left(B \sim 0.1 f_{0}[4]\right)$, the spectrum scaling can no longer be neglected, and the mutual uncertainty function does not provide reliable estimates of signals mutual time delay, due to the side maxima become comparable in magnitude with the main maximum.

To increase the efficiency of wideband signals mutual time delay estimating, a modified algorithm for calculating the mutual uncertainty function was proposed in [5]. The algorithm is based on the preselecting of narrow-band channels with a central frequency $f_{k}$ and spectral bandwidth $B_{k}$. The frequencies $f_{k}$ can correspond to the center frequencies of the physical frequency channels (for example in the case of frequency hopping signals [6]), but in the general case they can be arbitrary within the band of the received signals. It is advisable to choose the width of the spectral band of narrow-band channels so that the Doppler scaling of this spectral band is negligible for the application of the algorithm for calculating the mutual uncertainty function $\left(B_{k}<<f_{0}\right)$.

For the obtained narrow-band signals $S_{l, k}$ and $S_{2, k}$ an algorithm for calculating the mutual uncertainty function can be applied, since the effect of the spectrum scaling in the selected channels can be neglected.

It should be noted that the signals in the allocated narrow-band frequency channels are characterized by either a low transmission rate compared to the received signals (in the case of signals with OFDM modulation), or a short information part duration (in the case of frequency hopping signals [6]), in addition to low signal to noise ratio. The main maximum of the mutual uncertainty function of these signals will still be characterized by weak distinguishability. To increase the distinguishability of the main maximum, an algorithm is proposed based on averaging the "sections" of mutual uncertainty functions in narrow-band channels.

As a "section" of the mutual uncertainty function, we can consider a set of samples taken at a fixed value of the frequency shift, delivering a maximum to the absolute value of the mutual uncertainty function. In this case, two options are possible for summation the "sections" of the uncertainty function: coherent (summation of complex samples) and incoherent (summing the modules of samples).

In the case of coherent summation, the resulting distribution of the mutual uncertainty function values modules over the sections will take the form:

$$
\operatorname{CSS}(\tau)=\mid \sum_{k=1}^{M}\left[\left.\int_{-\infty}^{+\infty} s_{1, k}(t) \cdot s_{2, k}^{*}(t+\tau) \exp \left(-j 2 \pi \Delta f^{*} t\right) d t\right|_{\substack{\Delta f^{*}=\arg \max \left|A_{k}\right| \\ \Delta, \tau}}\right],
$$

where $k$ is the number of the frequency channel, $s_{k}(t)$ is the mutual uncertainty function section, $M$ is the number of frequency channels (and, accordingly, FIR filters), necessary to obtain a high distinguishability of the main maximum of the uncertainty function.

Similarly for incoherent summation:

$$
\operatorname{ISS}(\tau)=\sum_{k=1}^{M}\left|\int_{-\infty}^{+\infty} s_{1, k}(t) \cdot s_{2, k}^{*}(t+\tau) \exp \left(-j 2 \pi \Delta f^{*} t\right) d t\right| \underset{\substack{\Delta f^{*}=\arg \max \\ \Delta, \tau}\left|A_{k}\right|}{ } \mid .
$$


The disadvantage of this approach to constructing uncertainty function sections is the need for a full calculation of the body of uncertainty, which requires large amounts of memory. Another approach, requiring significantly smaller amounts of memory, is based on the formation of the mutual uncertainty function sections by sets of maximum values modules in the Fourier distribution for each value of the time shift between the signals. Similarly, the resulting distributions can be obtained using coherent and incoherent summation. In the case of coherent summation, the resulting distribution is defined as:

$$
\operatorname{CSMS}(\tau)=\left.\left|\sum_{k=1}^{M} \int_{-\infty}^{+\infty} s_{1, k}(t) \cdot s_{2, k}^{*}(t+\tau) \exp \left(-j 2 \pi \Delta f^{*} t\right) d t\right|\right|_{\Delta f^{*}=\arg \max \mid A_{k}(\tau, \Delta f)} \mid .
$$

In the case of incoherent summation:

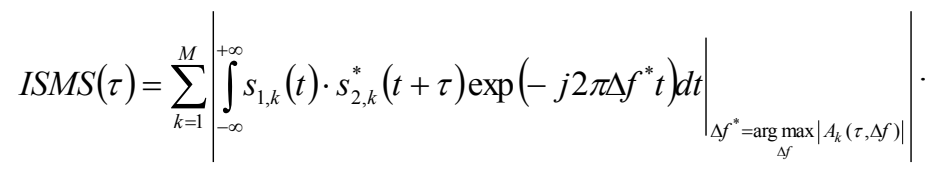
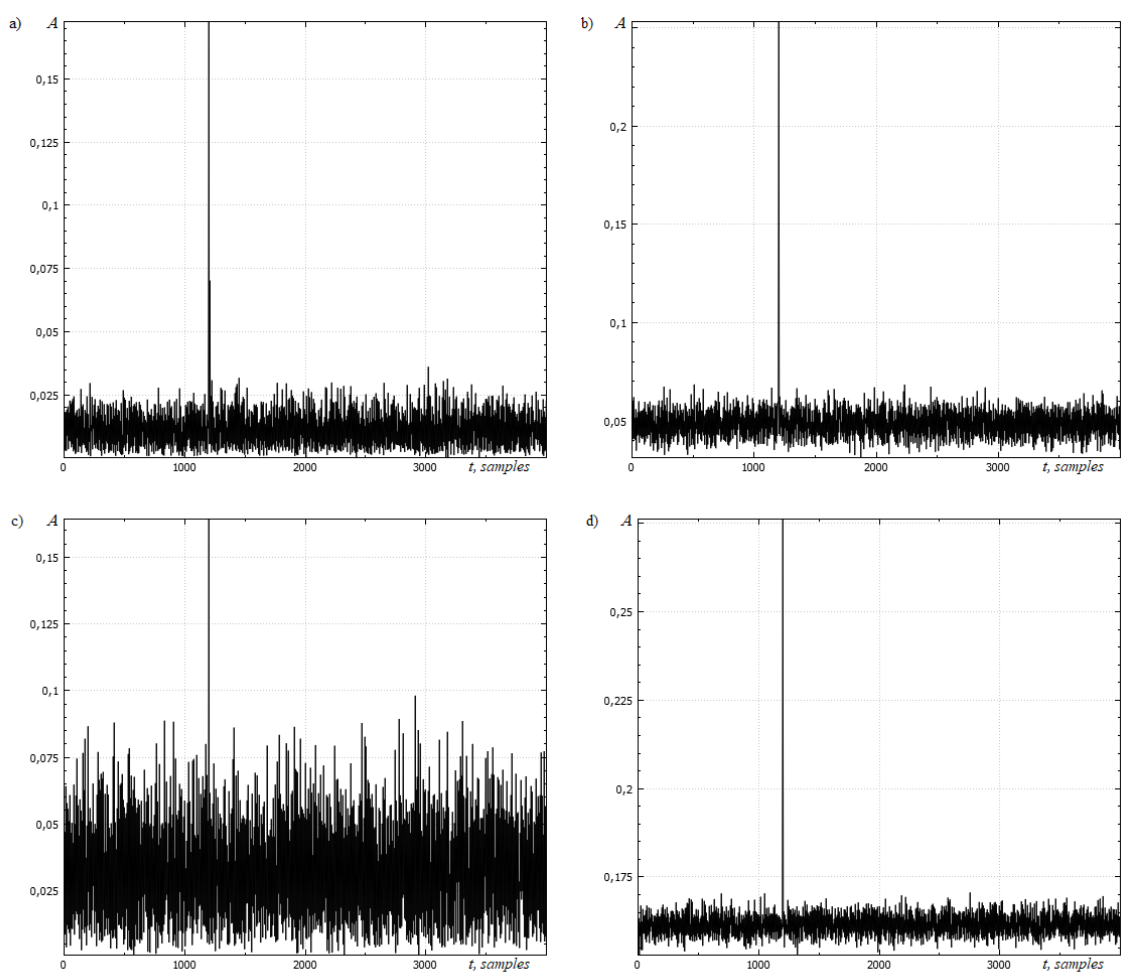

Fig. 1. The resulting uncertainty function sections a) CSS (5); b) ISS (6); c) CSMS (7); d) ISMS (8).

For the proposed algorithms, a study was made of the resulting distributions main maximum distinguishability obtained by expressions (3), (4), (5), (6). The study was performed for broadband OFDM signals (spectrum width - $420 \mathrm{MHz}$, minimum frequency $20 \mathrm{MHz}$, maximum frequency - $440 \mathrm{MHz}$, the number of frequency channels - 20, signalto-noise ratio $-5 \mathrm{~dB}$ ) (Figure 1). The distinguishability criterion of the main maximum can be estimated according to the expression: 


$$
K=\frac{\max \left(Q_{i}\right)-<Q>}{\sqrt{\frac{1}{N} \sum_{i=0}^{N-1}\left(Q_{i}-<Q>\right)^{2}}},
$$

where $N$ is the number of samples in the section, $Q$ is the resulting section.

Table 1 shows the values of the average distinguishability criterion (7) for the resulting functions constructed according to the considered algorithms.

Table 1. Average distinguishability criterion values.

\begin{tabular}{|c|c|}
\hline Algorithm & Criterion value \\
\hline CSS (3) & $21.9 \pm 1.5$ \\
\hline CSMS (5) & $8.0 \pm 1.5$ \\
\hline ISS (4) & $26.4 \pm 1.6$ \\
\hline ISMS (6) & $33.2 \pm 1.5$ \\
\hline
\end{tabular}

This study allows us to conclude that it is advisable to use the algorithm of averaging sections in the case of analysis of a variety of mutual uncertainty function with maximum of insufficient distinguishability. The summation of the complex values of the uncertainty function sections without taking into account additional information about the signals phase in the allocated narrow-band channels and taking into account the possible phase delays of the signals during band pass filtering does not give a noticeable improvement in the distinguishability of the main maximum. In addition, coherent summation imposes stringent requirements on the accuracy of compensation for the frequency shifts of the signal spectra.

Also a based on the proposed algorithms study of the correct mutual time delay determining probability dependence on the signal-to-noise ratio (SNR) was made. The probability of correct mutual time delay determining calculated based on statistical modeling. The decision threshold for signal detection (based on the distinguishability criterion (7)) was determined based on the Neumann-Pearson criterion. The results of the study are presented in Fig. 2.

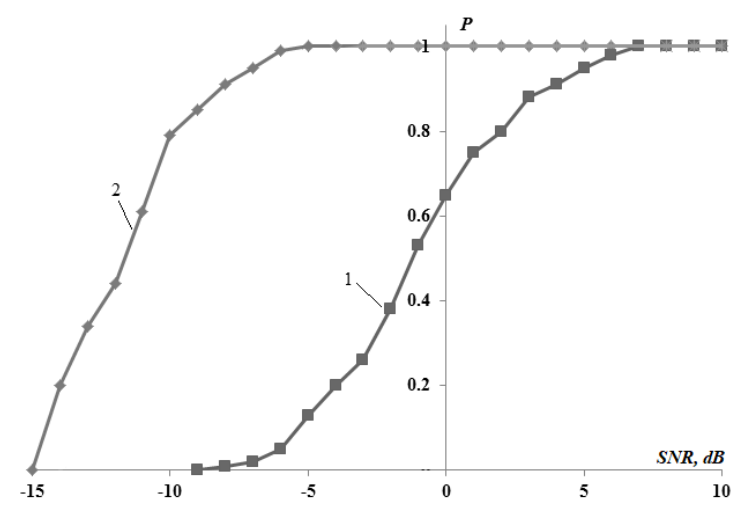

Fig. 2. The dependence of the correct time delay determining probability on the SNR:

1 - Mutual uncertainty function;

2 - Mutual uncertainty function with division into narrow-band channels (ISMS (6)). 
The obtained result indicates a significant improvement of the efficiency to signals with a small SNR (up to $-7 \mathrm{~dB}$ ) compared with the classical method of constructing and analyzing the mutual uncertainty function.

\section{References}

1. Y. P. Grishin, Y. M. Kazarinov, P. V. Ipatov, Radiotechnical systems (Vyssh. Schk. Publ., Moscow, 1990)

2. G. C. Carter, Coherence and Time Delay Estimation, Proceedings of IEEE, v. 75, issue 2, pp. 236-255 (1987)

3. S. Stein, Algorithms for Ambiguity Function Processing, IEEE Transactions on Acoustics, Speech and Signal Processing, v. ASSP-29, issue 3. pp. 588-599 (1981)

4. A. I. Perov, V. N. Kharisov, GLONASS. Principles of construction and operation (Radiotechnika Publ., Moscow, 2010)

5. R. A. Ershov, O. A. Morozov, V. R. Fidelman, Estimation of the mutual time delay of signals with pseudorandom frequency hopping, Radiophysics and Quantum Electronics, v. 58, issue 2 pp. 157-166 (2015)

6. I. V. Grin, R. A. Ershov, O. A. Morozov, Sistemy upravlenia i informacionnie tehnologii [Control Systems and Information Technology], v. 1, issue 3. pp. 18-22 (2015) 